\title{
Re-examining the Cairns-Tsallis model for ion acoustic solitons
}

\author{
G. Williams* and I. Kourakis \\ Centre for Plasma Physics, Department of Physics and Astronomy, Queen's University Belfast, BT7 INN Northern Ireland, United Kingdom \\ F. Verheest \\ Sterrenkundig Observatorium, Universiteit Gent, Krijgslaan 281, B-9000 Gent, Belgium \\ and School of Chemistry and Physics, University of KwaZulu-Natal, Durban 4000, South Africa \\ M. A. Hellberg \\ School of Chemistry and Physics, University of KwaZulu-Natal, Durban 4000, South Africa
}

(Received 30 March 2013; published 7 August 2013)

\begin{abstract}
Recently, a hybrid distribution function [Tribeche et al., Phys. Rev. E 85, 037401 (2012)] was proposed to describe a plasma species with an enhanced superthermal component. This combines a Cairns-type "nonthermal" form with the Tsallis theory for nonextensive thermodynamics. Using this alternative model, the propagation of arbitrary amplitude ion acoustic solitary waves in a two-component plasma is investigated. From a careful study of the distribution function it is found that the model itself is valid only for a very restricted range in the $q$-nonextensive parameter and the nonthermality parameter, $\alpha$. Solitary waves, the amplitude and nature of which depend sensitively on both $q$ and $\alpha$, can exist within a narrow range of allowable Mach numbers. Both positive and negative potential structures are found, and coexistence may occur.
\end{abstract}

DOI: 10.1103/PhysRevE.88.023103

PACS number(s): 52.35.Fp, 52.35.Mw, 52.35.Sb

\section{INTRODUCTION}

Nonlinear plasma dynamics is currently a very active topic of research, in which nonlinear plasma equations are analytically treated by a variety of methods [1] to gain deeper insight into the formation and behavior of structures such as solitary waves and shocks. The dynamics of ion acoustic waves have been studied for several decades, both theoretically [2-4] and experimentally [5-7].

It is by now established that the presence of energetic particles in plasmas, resulting in long-tailed distributions, is an intrinsic element in many space and laboratory observations. Different models have been proposed to describe this effect. We stress that these models do not address the actual mechanisms responsible for particle acceleration but rather model its effect on wave dynamics via phenomenological modification to the electron distribution function.

Cairns et al. [8] introduced a distribution designed to model enhanced high-energy tails that are frequently observed in space plasmas. It was expressed in terms of a parameter $\alpha$, which measures deviation from the Maxwellian distribution function, and showed that the nature of ion sound solitary structures can change in the presence of nonthermal electrons, producing nonlinear solitary waves that may have either enhanced or depleted density. Many analyses have subsequently used the Cairns model, for example, Refs. [3,9-15]. Verheest and Pillay [13] investigated large amplitude dust-acoustic solitary waves in plasmas with negatively charged cold dust and either nonthermally distributed ions or electrons. Verheest and Hellberg [14] examined large ion acoustic solitary waves and double layers in plasmas with positive ions and nonthermal electrons. Both investigations detailed the existence conditions necessary for such structures to be generated.

*gwilliams06@qub.ac.uk
A generalization of the Boltzmann-Gibbs-Shannon (BGS) entropic measure was proposed by Tsallis [16], in which a parameter $q$ characterizes the degree of nonextensivity of the system considered ( $q \rightarrow 1$ recovers the Maxwellian case). There are two separate physical regions, $-1<q \leqslant 1$, covering all velocities (and potentially representing highenergy tails) and $q \geqslant 1$, where the distribution function exhibits a thermal cut-off on the maximum value allowed for the velocity of the particles $[17,18]$. In recent years, this Tsallis model has frequently been used for investigations into ion acoustic dynamics in plasmas [19-26]. Using a pseudopotential approach, Dubinova and Dubinov [19] studied the dependence of the Mach number of ion acoustic solitons on the parameter $q$. Tribeche et al. [22] considered ion acoustic solitary wave generation in a two-component plasma with Tsallis-distributed electrons and found that their model allowed for both compressive and rarefactive solitons to arise.

It may be added for completeness that an alternative approach to non-Maxwellian plasma modeling is provided by the $\kappa$ distribution [27-29], which was introduced by Vasyliunas [27] to fit phenomenologically the power law-like dependence of electron distribution functions observed in space. It is, in fact, commonly fitted to observational data $[28,30]$. Interestingly, it has been argued that the $\kappa$ scenario derives from the Tsallis distribution [31,32], although this speculative analogy is rather phenomenological and remains the subject of debate $[28,32]$. We shall not pursue this analogy here as this is beyond our scope.

A recent study by Tribeche et al. [33] has proposed a hybrid Cairns-Tsallis distribution function, which purports to offer enhanced parametric flexibility in modeling nonthermal plasmas, as, in principle, such a two-parameter representation of the distribution function could be useful in fitting to a wider range of observed plasmas. Subsequently, Amour et al. [34] applied this distribution to the study of electron acoustic 
solitary waves in plasmas with an enhanced superthermal component. In this paper, we revisit the analysis of Ref. [33] by employing the Sagdeev pseudopotential method to investigate large-amplitude ion acoustic wave dynamics. We carefully analyze the range of validity of the model and present existence ranges for solitons supported by this distribution, arriving at very different conclusions from those reflected in Ref. [33].

The paper is structured as follows. In Sec. II, we critically examine the Cairns-Tsallis distribution function and place limits on its range of validity in general and specifically for application to soliton studies. This is followed in Sec. III by an outline of the fluid model adopted. Section IV deals with linear ion acoustic wave analysis, and arbitrary amplitude waves are discussed in Sec. V. In Secs. VI and VII, we obtain the lower and upper limits of the Mach speeds that can support solitons and double layers, and finally, we discuss our conclusions in Sec. VIII.

\section{THE CAIRNS-TSALLIS DISTRIBUTION AND ITS RANGE OF VALIDITY}

This paper revisits the analysis of Ref. [33] of ion acoustic solitary waves in a plasma with electrons having a CairnsTsallis distribution, but the physically realistic ranges of the parameters $q$ and $\alpha$ must be taken into account in order to do this effectively.

The one-dimensional hybrid Cairns-Tsallis distribution $[33,34]$ for the electrons is given by

$$
f_{\mathrm{CT}}=C_{q, \alpha}\left(1+\alpha \frac{v_{x}^{4}}{v_{t e}^{4}}\right)\left[1-(q-1) \frac{v_{x}^{2}}{2 v_{t e}^{2}}\right]^{1 /(q-1)},
$$

where $v_{t e}=\left(T_{e} / m_{e}\right)^{1 / 2}$ is the electron thermal velocity and $q$ and $\alpha$ are real parameters, associated with the so-called nonextensivity [16] and the number of nonthermal electrons [8] in the distribution, respectively. Furthermore, $C_{q, \alpha}$ is a constant of normalization, which is dependent on $q$ and $\alpha$, and is given explicitly by Refs. $[33,34]$ as

$$
\begin{aligned}
& C_{q, \alpha} \\
& =\left\{\begin{array}{c}
n_{e 0} \sqrt{\frac{m_{e}}{2 \pi T_{e}}} \frac{\Gamma\left(\frac{1}{1-q}\right)(1-q)^{5 / 2}}{\Gamma\left(\frac{1}{1-q}-\frac{5}{2}\right)\left[3 \alpha+\left(\frac{1}{1-q}-\frac{3}{2}\right)\left(\frac{1}{1-q}-\frac{5}{2}\right)(1-q)^{2}\right]} \\
\text { for }-1<q \leqslant 1, \\
n_{e 0 \sqrt{\frac{m_{e}}{2 \pi T_{e}}}} \frac{\Gamma\left(\frac{1}{q-1}+\frac{3}{2}\right)(q-1)^{5 / 2}\left(\frac{1}{q-1}+\frac{3}{2}\right)\left(\frac{1}{q-1}+\frac{5}{2}\right)}{\Gamma\left(\frac{1}{q-1}+1\right)\left[3 \alpha+\left(\frac{1}{q-1}+\frac{3}{2}\right)\left(\frac{1}{q-1}+\frac{5}{2}\right)(q-1)^{2}\right]} \\
\text { for } q \geqslant 1 .
\end{array}\right.
\end{aligned}
$$

For $q \rightarrow 1$, both expressions recover the Cairns distribution. These coefficients are, of course, found by evaluating the lowest moment of $f_{\mathrm{CT}}$ in each of the two ranges of $q$. However, it turns out that, unlike the situation for the pure Tsallis distribution, the integral to obtain the upper expression is not valid over the whole range $-1<q \leqslant 1$, and we shall discuss the implications in more detail below.

For a start, we reiterate that the reason for considering the Cairns-Tsallis distribution is to study the effects of the superthermal particles in the non-Maxwellian tail of the distribution. We note also that the distribution in Eq. (1) is specifically a product of the Cairns and the Tsallis distribution functions.

It is well-known that the pure Tsallis distribution function behaves very differently in the two ranges, $-1<q<1$ and $q>1[17,18]$. In the former range, the distribution is nonzero over the full range of velocities from $-\infty$ to $+\infty$, and it reflects an excess of superthermal particles in the non-Maxwellian tail. It is, thus, in principle, similar to a power-law or a $\kappa$ distribution in that sense. On the other hand, for $q>1$, it exhibits a thermal cutoff, is zero in the region $|v|>\left(T_{e} / m_{e}\right)^{1 / 2}$, and is, thus, bounded by the thermal velocity. Hence, it follows that the product Cairns-Tsallis distribution, too, is zero beyond the thermal speed, and in that $q$ range is not of any interest for the representation of the commonly observed non-Maxwellian tail distributions.

Unlike Refs. [33,34], we shall in this work not consider values of $q>1$, as this physical region is not consistent with our focus on a long-tailed distribution function associated with an excess of energetic particles and is physically irrelevant for our purposes.

However, we do point out that if one were to consider the range $q>1$, a separate expression for the electron number density would be required from that given for $-1<q \leqslant 1$ $[33,34]$ in a way similar to that found, for instance, in Eqs. (9) and (10) of Ref. [26]. This applies also for $\alpha=0$ (pure Tsallis distribution), and hence, it is surprising that several previous analyses involving pure Tsallis-type distributions (i.e., for $\alpha=0$ ) $[22,24,25]$ use the same electron number density expressions for values of $q$ both above and below 1 .

We now return to the expression for $f_{\mathrm{CT}}$ and consider its behavior for large $|v|$, where $v=v_{x} / v_{t e}$. In that range, $f_{\mathrm{CT}} \propto$ $(1 / 2) \alpha(1-q) v^{4+(2 /[q-1])}$, that is, $f_{\mathrm{CT}} \propto v^{(4 q-2) /(q-1)}$. Clearly, for any physically realistic distribution function, a minimum requirement is that the function itself should not diverge as $|v| \rightarrow \infty$. That leads to a minimum value of $q=1 / 2$.

However, to obtain physically realistic integrals, one obviously needs to satisfy the more stringent requirement that $f_{\mathrm{CT}} \rightarrow 0$ faster than $|v|^{-1}$. It follows that the expression for $f_{\text {CT }}$ is valid only for $q>3 / 5$. We note that, in fact, the integral diverges for $q=3 / 5$ [37].

Indeed, if one indiscriminately uses the coefficients, $C_{q, \alpha}$ of Refs. [33,34] in the invalid range, one finds, for instance, that at $q=3 / 5, C_{q, \alpha}$, and hence, also $f_{\mathrm{CT}}$ and the electron density, vanishes, while for the range $1 / 3<q<3 / 5$, they are negative. It is, thus, imperative that one carefully analyzes the behavior of the distribution function rather than blindly applying it.

We note that this behavior, for $\alpha \neq 0$, is different from that of the standard Tsallis distribution function for which $f_{T} \propto v^{2 /(q-1)}$ for $|v| \rightarrow \infty$, and which is well-behaved and normalizable for $-1<q \leqslant 1$.

In view of the above arguments, this study is restricted to values of $q$ within the very limited range, $0.6<q \leqslant 1$. This is in contrast to Ref. [33], who apply the model to both $-1<q \leqslant 1$ and $q \geqslant 1$.

Furthermore, following Ref. [33], we shall discuss ion acoustic solitary waves in a plasma with electrons having a Cairns-Tsallis distribution. 

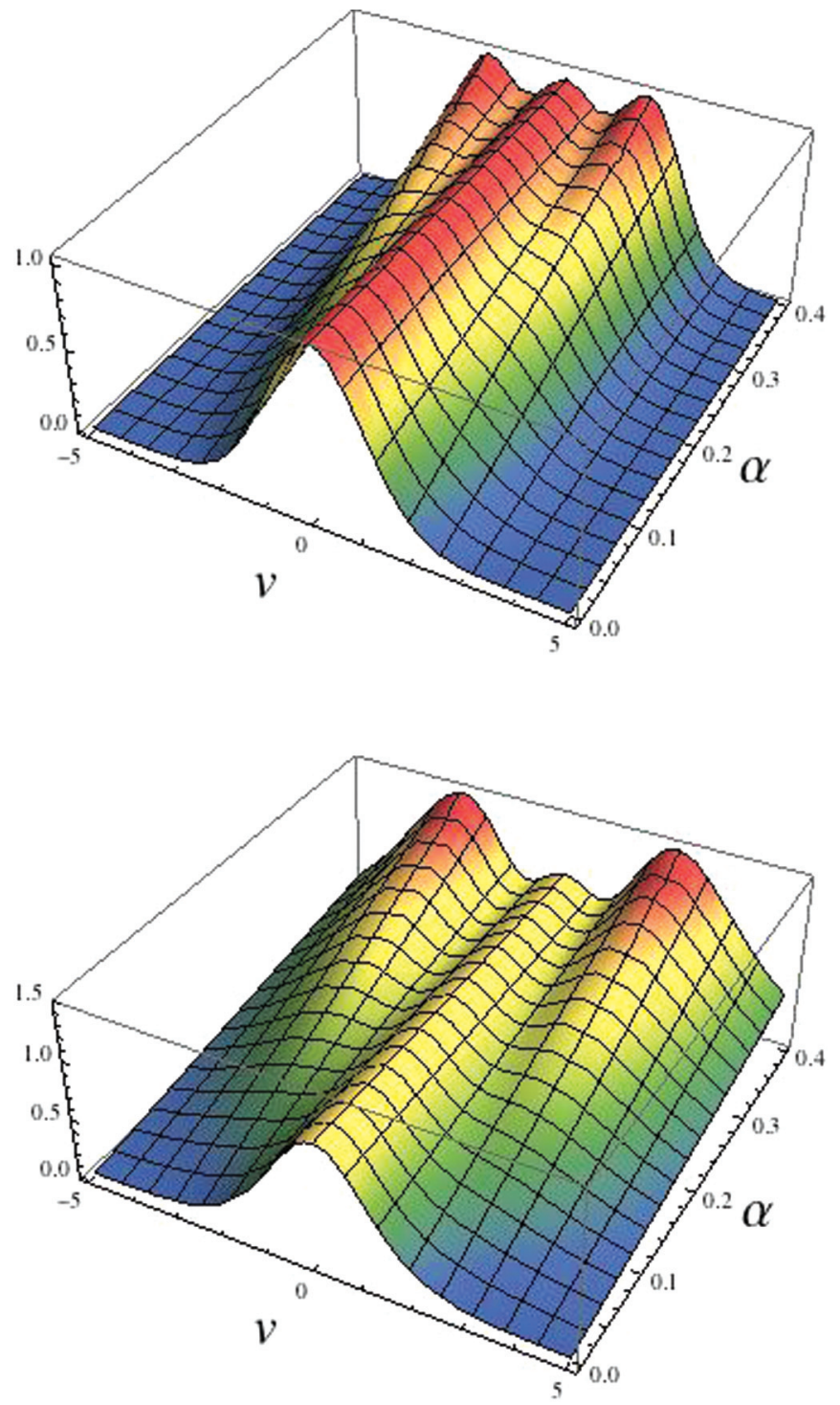

FIG. 1. (Color online) 3D Plot of the Cairns-Tsallis distribution function with $q \rightarrow 1$ (pure Cairns distribution) above, and with $q=$ 0.8 below. For simplicity, here, we take $v=v_{x} / v_{t e}$.

Now, it has been noted by Refs. [13] and [14] that at values of $\alpha$ larger than $\alpha \simeq 0.25$, the pure Cairns distribution function $(q=1)$ presents nonmonotonic behavior as it develops sidewings, possibly leading to a kinetic instability. One would not expect stable nonlinear structures such as solitons to be supported by such a linearly unstable situation. That implies a need to introduce a cutoff in $\alpha$ governed by this consideration.

This nonmonotonic shape can be seen in Fig. 1, where the pure Cairns distribution $(q=1)$ in the upper figure shows subsidiary maxima for $\alpha>0.25$. In the lower figure, the Cairns-Tsallis distribution for $q=0.8$ is presented, and this shows that monotonicity breaks down already at about $\alpha=0.15$.

It follows that there is a $q$-dependent limit on $\alpha$ for the monotonicity of the distribution function to be preserved. This is investigated in Figs. 2 and 3.

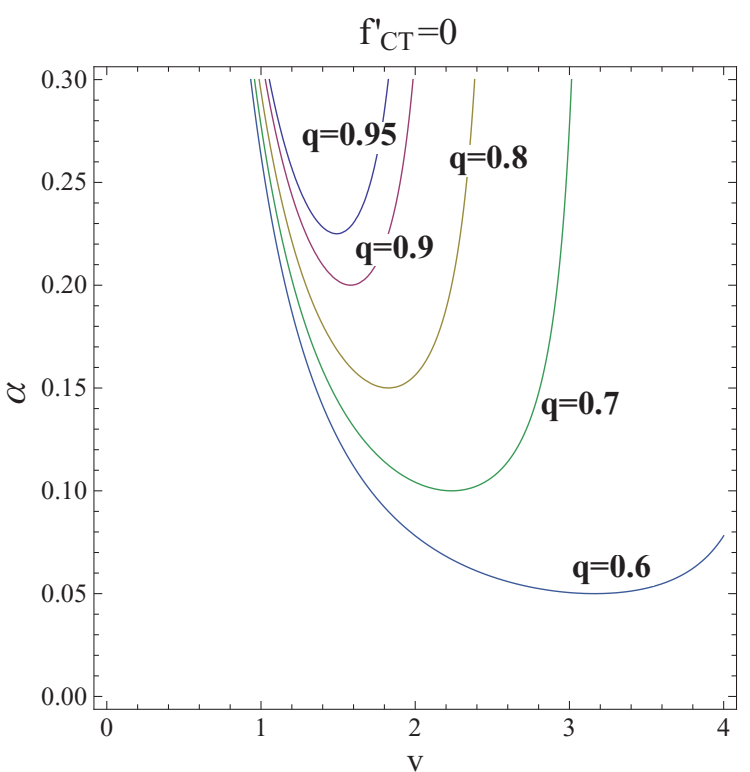

FIG. 2. (Color online) Contour plot of the derivative of the CairnsTsallis distribution function set equal to zero, for $q=0.6,0.7,0.8,0.9$, and 0.95 as labeled. For simplicity, here, we take $v=v_{x} / v_{t e}$.

Clearly, the limiting values of $(q, \alpha)$ occur at values given by the roots of the equation

$$
\partial f_{\mathrm{CT}}\left(v_{x} ; q, \alpha\right) / \partial v_{x}=0 .
$$

The derivative of the Cairns-Tsallis distribution function is given by

$$
\begin{aligned}
\frac{\partial f_{\mathrm{CT}}}{\partial v_{x}}= & C_{q, \alpha} \frac{2 v_{x}\left(1-[q-1] \frac{v_{x}^{2}}{2 v_{t e}^{2}}\right)^{1 /(q-1)}}{v_{t e}\left(-2+[q-1] \frac{v_{x}^{2}}{v_{t e}^{2}}\right)} \\
& \times\left(\frac{v_{x}^{4}}{v_{t e}^{4}}(\alpha[2 q-1])-4 \alpha \frac{v_{x}^{2}}{v_{t e}^{2}}+1\right) .
\end{aligned}
$$

Figure 2 is a contour plot of the limit of the monotonic distribution function range, given by $f_{\mathrm{CT}}^{\prime}=0$, for some typical values of $q$ within the narrow allowed range discussed above. It can be seen that for $q=0.95$, the monotonicity is preserved

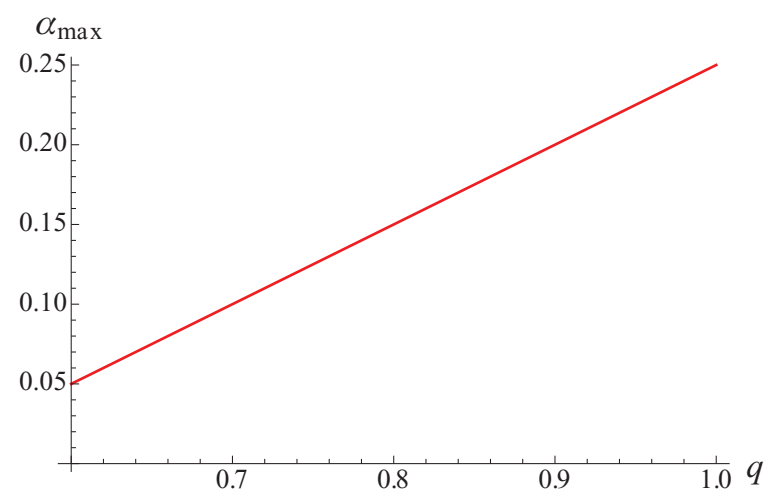

FIG. 3. (Color online) A plot showing the maximum value of $\alpha$ allowed for corresponding values of $q$ for monotonicity to be preserved. The linear relation is $\alpha=(2 q-1) / 4$, which satisfies $f_{\mathrm{CT}}^{\prime}(v)=0$; see Eq. (4). 
up to $\alpha \simeq 0.22$, while for the lower limit of $q$, namely, $q=0.6$, the distribution function shows subsidiary maxima already at about $\alpha \simeq 0.05$.

Solving $f_{\mathrm{CT}}^{\prime}(v)=0$ for $v_{x}^{2} / v_{t e}^{2}$ to determine the existence conditions for real roots involves the discriminant $4 \alpha-2 q+$ 1 , which in turn yields the crossover value of $\alpha=(2 q-1)$ / 4 , always, of course, within the range $0.6<q \leqslant 1$. This information is summarized in Fig. 3.

With the above considerations in mind, we restrict our analysis below to the ranges $0 \leqslant \alpha<0.25$, and $0.6<q \leqslant 1$, subject to the physical cutoff imposed by the monotonicity condition, $\alpha=(2 q-1) / 4$.

\section{THE FLUID MODEL}

We shall now consider ion acoustic waves propagating in one-dimensional plane geometry in an unmagnetized plasma consisting of cold ions $\left(T_{i}=0\right)$ and electrons with a CairnsTsallis distribution function. As is usual for ion-acoustic structures, we require that the wave phase speed lies between the ion and electron thermal speeds, that is, $v_{t i} \ll v_{p h} \ll v_{t e}$, to avoid Landau damping [35]. From the second inequality it follows that, on the ion timescale, the electrons can reach an equilibrium at every stage of the wave development. Thus, in this approach, the electron inertia is effectively neglected $[35,36]$, and the electrons are often described as being massless [4].

We employ the following normalized (dimensionless) system of equations for the ion fluid:

$$
\begin{aligned}
\frac{\partial n}{\partial t}+\frac{\partial(n u)}{\partial x} & =0 \\
\frac{\partial u}{\partial t}+u \frac{\partial u}{\partial x} & =-\frac{\partial \phi}{\partial x}, \\
\frac{\partial^{2} \phi}{\partial x^{2}} & =n_{e}-n,
\end{aligned}
$$

where $n, \phi$, and $u$ represent the ion density (normalized to the equilibrium ion density $n_{i 0}$ ), the electrostatic potential (normalized by $T_{e} / e$ ), and the ion velocity (normalized to the speed of ion sound waves in a simple Maxwellian electronion plasma, $c_{s}=\left(T_{e} / m_{i}\right)^{1 / 2}$, that is, neglecting the nonMaxwellian effects inherent in the Cairns-Tsallis distribution). The ion subscript " $i$ " is omitted in the system above, where obvious. Time has been normalized to the inverse of the ion plasma frequency $\left(\omega_{p i}^{-1}=\left[m_{i} \epsilon_{0} / e^{2} n_{i 0}\right]^{1 / 2}\right)$, and length to the Debye length $\left(\lambda_{D}=\left[\epsilon_{0} T_{e} / e^{2} n_{e 0}\right]^{1 / 2}\right)$. As is customary, $m_{i}$ represents the ion mass, $e$ the electron charge, and $T_{e}$ the electron kinetic temperature (in energy units). The ionic charge state is assumed to be $Z_{i}=1$ for simplicity, and ion thermal effects have been neglected in this model. We have assumed that at equilibrium the electron and ion densities are equal, i.e., $n_{e 0}=n_{i 0}$.

To derive the number density expression for the electron fluid in this case, one turns to the distribution function, $f_{\mathrm{CT}}$. To account for the effect of an unnormalized electrostatic potential, $\varphi$, one replaces $v_{x}^{2}$ by $\left(v_{x}^{2}-\left[2 e \varphi / m_{e}\right]\right)$ in Eq. (1), using the expression for $C_{q, \alpha}$ as given by Eq. (2), but for the range $0.6<q \leqslant 1$ only. Integrating over all velocities in the usual way and normalizing, one obtains the normalized electron density, $n_{e}(\phi)$, in the presence of a normalized electrostatic potential, $\phi$.

This leads to the same form as found earlier [33,34],

$$
n_{e}=[1+(q-1) \phi]^{[1 /(q-1)]+1 / 2}\left(1+A \phi+B \phi^{2}\right),
$$

where

$$
\begin{gathered}
A=-\frac{16 q \alpha}{3-14 q+15 q^{2}+12 \alpha} \\
B=\frac{16(2 q-1) q \alpha}{3-14 q+15 q^{2}+12 \alpha} .
\end{gathered}
$$

However, we recall that we are using this expression only for the range $0.6<q \leqslant 1$. It is straightforward to show that the Maxwell-Boltzmann distribution is recovered in the limit $\alpha=0$, as $q \rightarrow 1$

\section{LINEAR WAVE ANALYSIS}

Following Ref. [33], we can linearize the system of fluid equations in the usual way to obtain the linear dispersion equation:

$$
\omega^{2}=\frac{k^{2}}{k^{2}+c_{1}},
$$

where

$$
c_{1}=\frac{1}{2}(1+q+2 A),
$$

and $A$ is as defined in Eq. (8). Comparing this result with that of Tribeche et al. [33], it is found that there is agreement with Eq. (9) therein, that is, $c_{1} \equiv k_{D, q, \alpha}^{2}$.

On the other hand, we note that there appears to be an error in the denominator of their Eq. (8), where they have written

$$
\omega^{2}=\frac{k^{2}}{k^{2}+k_{D, q, \alpha}} .
$$

As a matter of fact, a similar error reappears in Ref. [34] by the same authors.

From Eq. (9), we note that $c_{1}$ is essentially the effective inverse (square) screening length, that is, $\lambda_{D}=c_{1}^{-1 / 2}$. Importantly, this now depends on $q$ and $\alpha$. This reflects the fact that energetic particles affect the Debye screening mechanism by modifying the electron cloud distribution surrounding the ions, as is well-known for waves in a $\kappa$-distributed plasma [28]. For the sake of rigor, we emphasize that $c_{1}^{-1 / 2}$ is also related to the true sound speed in the given plasma configuration, as will be discussed below.

\section{ARBITRARY AMPLITUDE SOLITARY WAVE THEORY}

We anticipate the existence of arbitrary amplitude traveling solitary waves (that is, waves which have a constant profile and shape when viewed in a frame moving with the excitation), by assuming that all the fluid variables in the evolution equations depend on a single variable, $X=x-M t$, where $M$, known as the "Mach number," is the speed of the soliton normalized by $c_{s}$. We emphasize for rigor that, as we shall show below, the "true" sound speed is given by Eq. (22) and is, thus, not $c_{s}$. In this sense, the true Mach number is actually $M / M_{\min }$ [ $M_{\min }$ to be defined by Eq. (22) below]. Following Ref. [33], 
and using the Sagdeev pseudopotential method [2], the transformed variable is incorporated into our system of fluid equations above to give

$$
\begin{gathered}
-M \frac{\partial n}{\partial X}+\frac{\partial(n u)}{\partial X}=0, \\
-M \frac{\partial u}{\partial X}+u \frac{\partial u}{\partial X}=-\frac{\partial \phi}{\partial X}, \\
\frac{\partial^{2} \phi}{\partial X^{2}}=(1+(q-1) \phi)^{\left(\frac{1}{q-1}\right)+\frac{1}{2}}\left(1+A \phi+B \phi^{2}\right)-n .
\end{gathered}
$$

Integrating Eqs. (11) and (12) and applying boundary conditions for localized perturbations $(n \rightarrow 1, u \rightarrow 0, \phi \rightarrow 0$, as $X \rightarrow \pm \infty)$, we find

$$
n=\frac{1}{\left(1-\frac{2 \phi}{M^{2}}\right)^{\frac{1}{2}}} .
$$

Note here that for $n$ to be real, one requires that $M^{2} \geqslant 2 \phi$. This reality condition is always satisfied for negative values of $\phi$, and hence, negative potential pulses are never to be excluded a priori (provided that the Mach number exceeds a minimum allowed value, which we discuss below).

We now substitute Eq. (14) into Poisson's Eq. (13), multiply the resulting equation by $\frac{\partial \phi}{\partial X}$, integrate and apply the boundary condition $\frac{\partial \phi}{\partial X} \rightarrow 0$, as $X \rightarrow \pm \infty$. So, finally, we see that

$$
\frac{1}{2}\left(\frac{\partial \phi}{\partial X}\right)^{2}+\Psi(\phi, M)=0
$$

where $\Psi(\phi, M)$ is the Sagdeev pseudopotential, expressed as

$$
\begin{aligned}
\Psi(\phi, M)= & M^{2}\left[1-\left(1-2 \phi / M^{2}\right)^{\frac{1}{2}}\right] \\
& +\frac{2\left\{F-[1+(q-1) \phi]^{\frac{3 q-1}{2 q-2}}\left(D \phi^{2}+E \phi+F\right)\right\}}{(7 q-5)\left(3+12 \alpha-14 q+15 q^{2}\right)},
\end{aligned}
$$

where $D=32 \alpha q^{2}-16 \alpha q, E=-48 \alpha q$, and $F=60 \alpha+$ $35 q^{2}-46 q+15$.

It should be noted that this result is not in agreement with Eq. (11) of Ref. [33], which is shown below for completeness:

$$
\begin{aligned}
V(\Psi)= & \frac{[1+(q-1) \Psi]^{1 /(q-1)+3 / 2}}{(q-1)\left(\frac{1}{q-1}+\frac{3}{2}\right)}\left(-1+A \Psi-B \Psi^{2}\right) \\
& +\frac{[1+(q-1) \Psi]^{1 /(q-1)+5 / 2}}{(q-1)^{2}\left(\frac{1}{q-1}+\frac{3}{2}\right)\left(\frac{1}{q-1}+\frac{5}{2}\right)}(A+2 B \Psi) \\
& +\frac{2 B\left\{1-[1+(q-1) \Psi]^{1 /(q-1)+7 / 2}\right\}}{(q-1)^{3}\left(\frac{1}{q-1}+\frac{3}{2}\right)\left(\frac{1}{q-1}+\frac{5}{2}\right)\left(\frac{1}{q-1}+\frac{7}{2}\right)} \\
& +\frac{\left[1-\frac{A}{(q-1)\left(\frac{1}{q-1}+\frac{5}{2}\right)}\right]}{(q-1)\left(\frac{1}{q-1}+\frac{3}{2}\right)}+M^{2}\left[1-\left(1-\frac{2 \Psi}{M^{2}}\right)^{1 / 2}\right] .
\end{aligned}
$$

We have examined the limiting cases of the pseudopotentials above to investigate this discrepancy and have found that in the limit $\alpha \rightarrow 0$, both Eqs. (16) and (17) recover the Tsallis pseudopotential, in agreement with Ref. [22], Eq. (14) therein:

$$
\begin{aligned}
\Psi(\phi, M)_{\mid \alpha=0}= & M^{2}\left[1-\left(1-2 \phi / M^{2}\right)^{\frac{1}{2}}\right] \\
& +\frac{2\left[1-(1+(q-1) \phi)^{\frac{3 q-1}{2 q-2}}\right]}{3 q-1} .
\end{aligned}
$$

The limiting case whereby $q \rightarrow 1$ and $\alpha \rightarrow 0$ yields the wellknown Maxwellian pseudopotential [2,4] for both Eqs. (16) and (17).

$\Psi(\phi, M)_{\mid \alpha=0, q \rightarrow 1}=1-e^{\phi}+M^{2}\left[1-\left(1-2 \phi / M^{2}\right)^{\frac{1}{2}}\right]$.

In the extensive limiting case $(q \rightarrow 1)$, Eq. (16) recovers the Cairns pseudopotential [in agreement with Eq. (5) from Verheest and Hellberg's study [14] if we substitute $\beta=$ $4 \alpha /(1+3 \alpha)$ therein]:

$$
\begin{aligned}
\Psi(\phi, M)_{\mid(q \rightarrow 1)}= & M^{2}\left[1-\left(1-2 \phi / M^{2}\right)^{\frac{1}{2}}\right] \\
& +\frac{1+15 \alpha-e^{\phi}\left(4 \alpha \phi^{2}-12 \alpha \phi+15 \alpha+1\right)}{1+3 \alpha} .
\end{aligned}
$$

However, we have not been able to recover the Cairns pseudopotential [14] from the Tribeche et al. [33] expression shown in Eq. (17), and so we must conclude that their calculation appears to be flawed.

We have depicted the Sagdeev pseudopotential function for some small variations of $\alpha$, with $q=0.8$ and a Mach number $M=1.4$. The result is shown in Fig. 4. As can be seen therein, at $\alpha=0.0617$, there is a small positive pseudopotential well, but no negative counterpart. As the value of $\alpha$ is increased incrementally to 0.0619 , we can see a negative double layer coexisting with a positive potential well. At larger values of $\alpha$, we see more evidence of this coexistence, in this case of negative and positive solitons, with potential wells shrinking in both width and depth. Note that by coexistence we mean that either a negative or positive solitary wave is possible for these parameter values.

Figure 5 shows the resulting solitary wave pulses, on integration of the pseudopotential wells above. It is seen that the negative pulses are of higher amplitude than the positive pulses but gradually diminish as the value of $\alpha$ is increased.

\section{LOWER LIMIT OF MACH NUMBER $M$ (TRUE ACOUSTIC SPEED)}

In order for the anticipated type of motion to be possible (i.e., positive potential solitons moving at normalized velocity $M)$, the standard Sagdeev requirement has been that the potential $\Psi(\phi, M)$ must possess a maximum at the origin $\phi=0$ and must also have a root at $\phi_{m} \neq 0$. More recent work has shown that a finite soliton can also exist if the function has a point of inflexion at the origin [14,15], yielding a more general requirement that $\Psi$ should not have a strict minimum at the origin.

At the lower limit of the Mach number $M$, we thus require that $\Psi^{\prime \prime}(\phi, M)_{\mid \phi=0} \leqslant 0$.

At $\phi=0$,

$$
\Psi^{\prime \prime}(0, M)=-\frac{1}{2}-\frac{1}{2} q+\frac{16 q \alpha}{3+12 \alpha-14 q+15 q^{2}}+\frac{1}{M^{2}} .
$$



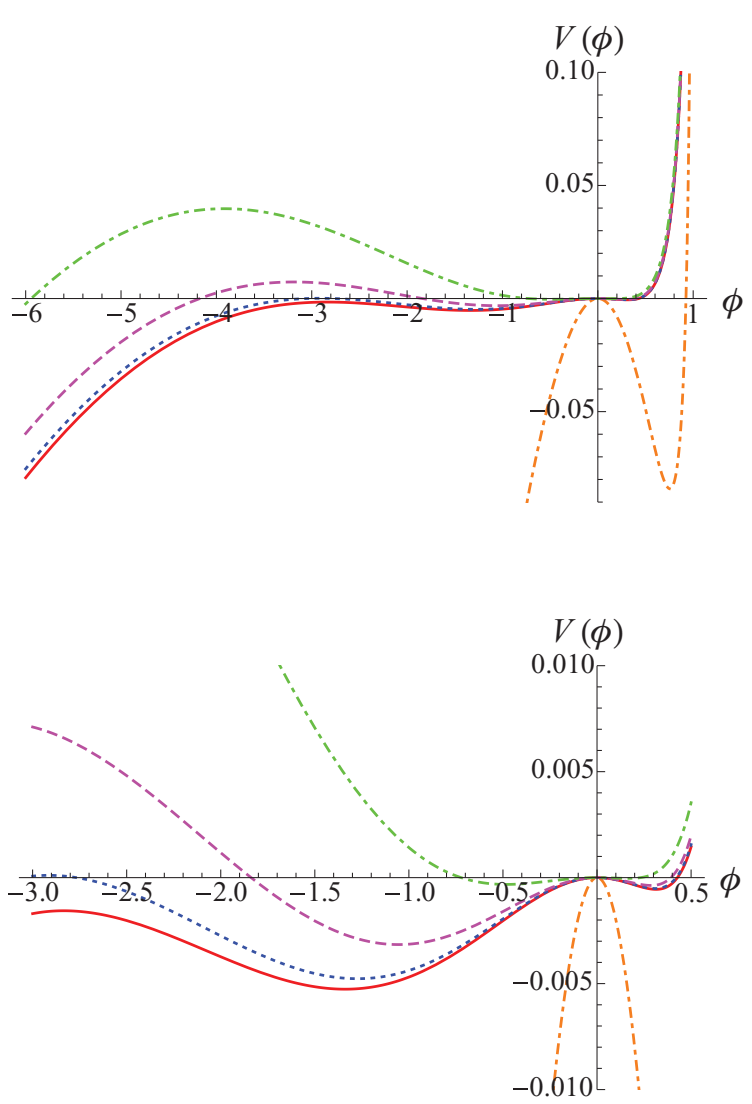

FIG. 4. (Color online) Upper plot: the Sagdeev pseudopotential function, with $q=0.8, M=1.4, \alpha=0$ (orange dot-dashed line), 0.0617 (red continuous line), 0.0619 (blue dotted line), 0.0621 (magenta dashed line), and 0.0657 (green dot-dashed line). Lower plot shows the same function, zoomed in so that we see the pseudopotential wells more clearly.

Note that this can be cast in the form

$$
\Psi^{\prime \prime}(0, M)=-c_{1}+\frac{1}{M^{2}} \leqslant 0,
$$

where $c_{1}$ is given by Eq. (10) above. This places a lower limit on $M\left(M_{\min }\right)$, so that $M_{\min }=1 / \sqrt{c_{1}}$, in our dimensionless

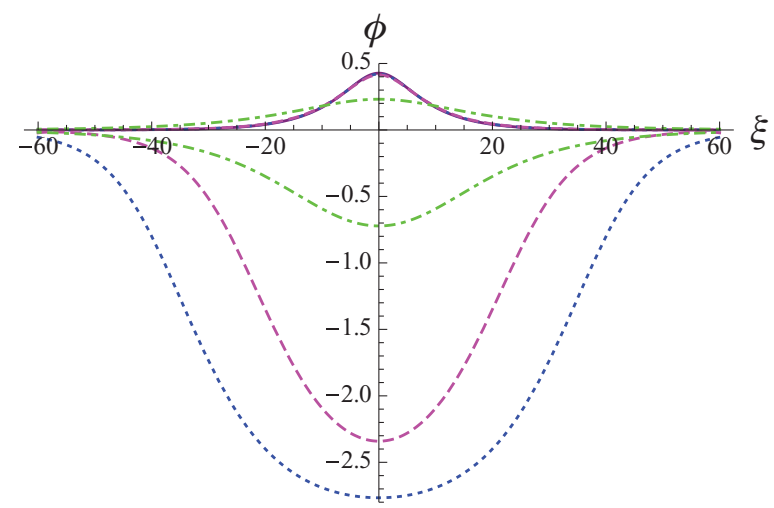

FIG. 5. (Color online) Plot of the soliton pulses arising from the values $q=0.8, M=1.4, \alpha=0.0617$ (red continuous line), 0.0619 (blue dotted lines), 0.0621 (magenta dashed lines), and 0.0657 (green dot-dashed lines).

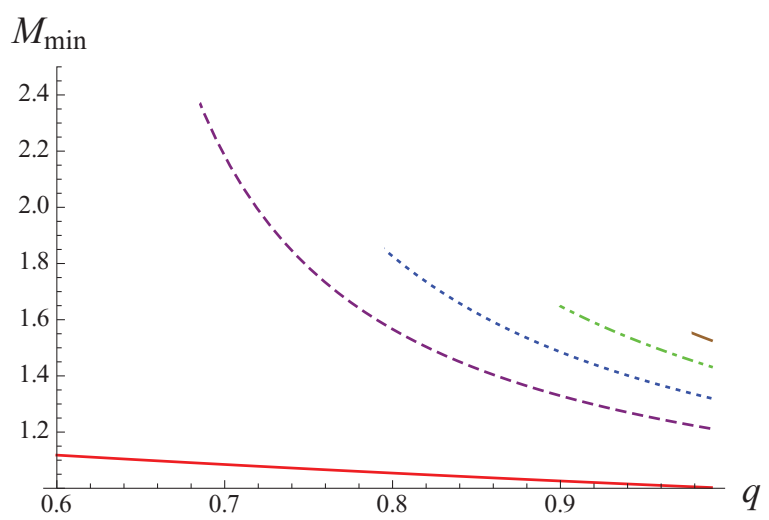

FIG. 6. (Color online) Plot of the minimum Mach number against the nonextensivity parameter $q$ for $\alpha=0$ (red continuous line), $\alpha=$ 0.1 (purple dashed line), $\alpha=0.15$ (blue dotted line), $\alpha=0.2$ (green dot-dashed line), and $\alpha=0.24$ (brown continuous line, just visible) in the range $0.6<q \leqslant 1$.

equation format, that is

$$
M \geqslant M_{\min }=\frac{1}{\sqrt{c_{1}}},
$$

which is exactly the acoustic speed $\lim _{k \rightarrow 0}(\omega / k)$, as prescribed from Eq. (9).

Using the expressions from Eqs. (10) and (8), one finds that the true normalized ion acoustic phase velocity in a plasma whose electrons have a Cairns-Tsallis distribution, satisfies a function of $q$ and $\alpha$, given by

$$
\begin{aligned}
v_{p h}^{2} & =\frac{1}{\frac{1}{2}(1+q+2 A)} \\
& =\frac{6-28 q+30 q^{2}+24 \alpha}{3-11 q+q^{2}+15 q^{3}-20 q \alpha+12 \alpha} .
\end{aligned}
$$

The above expression for $M_{\min }$ does not agree with the value for $M_{\min }$ found in Ref. [33], Eq. (12).

However, we have checked the limits of the expression for the Mach number $M_{\min }$ above to compare with external findings. The limit of Eq. (22), as $\alpha \rightarrow 0$ (the Tsallis limit), is found to be $M_{\min }=\sqrt{2 /(q+1)}$, which agrees with Ref. [22],

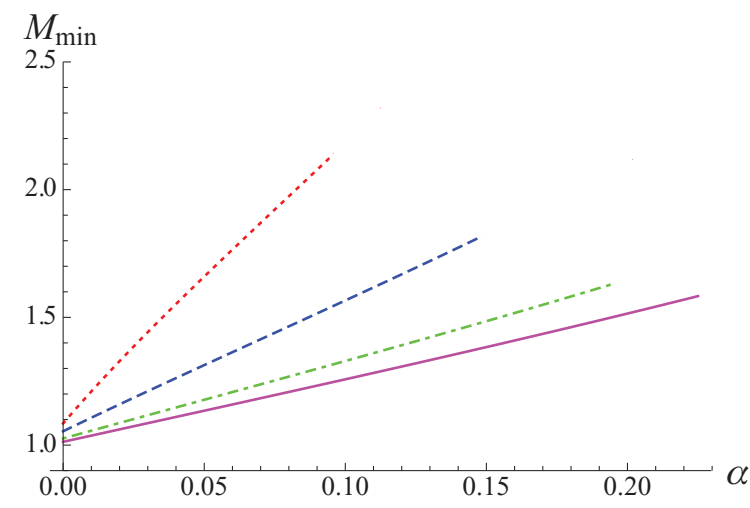

FIG. 7. (Color online) Plot of the minimum Mach number against the parameter $\alpha$ for $q=0.7$ (red dotted line), $q=0.8$ (blue dashed line), $q=0.9$ (green dot-dashed line), and $q=0.95$ (magenta continuous line). 


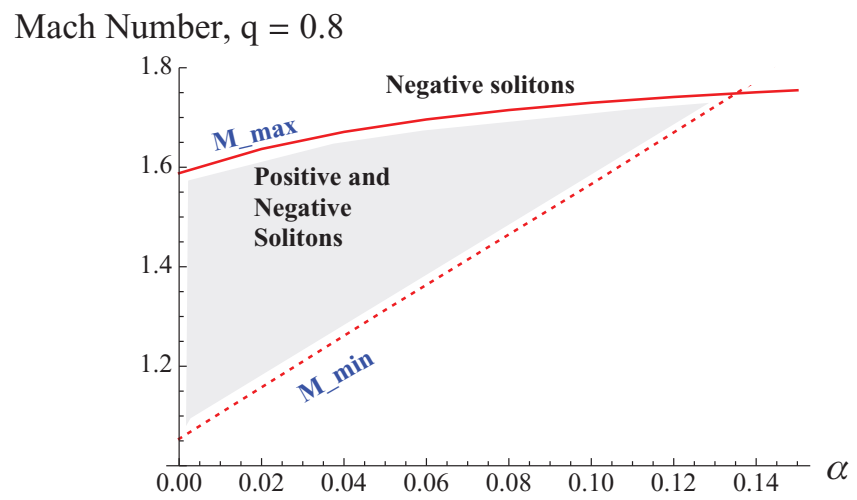

Mach Number, $q=0.9$

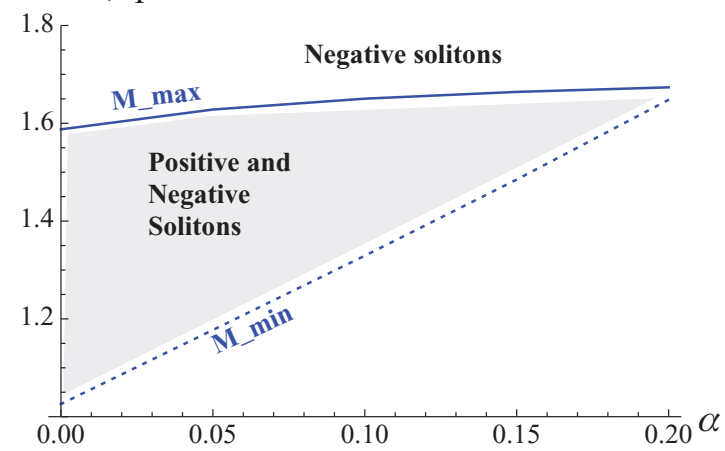

Mach Number, $q=0.95$

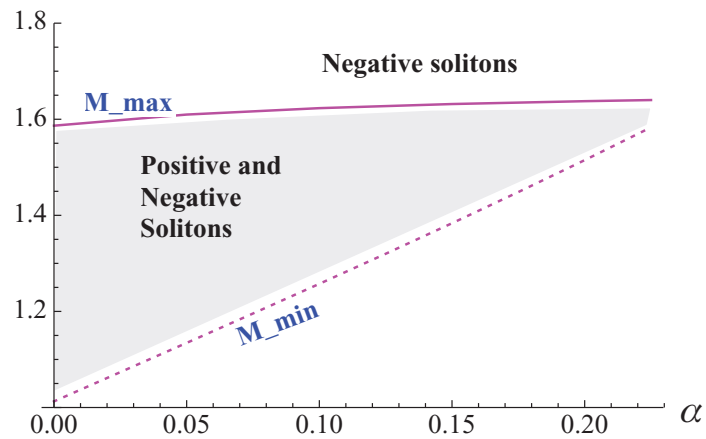

FIG. 8. (Color online) Plot of the maximum (solid lines) and minimum Mach number (dotted lines) against the nonthermality parameter $\alpha$, for $q=0.8$ in the range $0 \leqslant \alpha \leqslant 0.15$ (top plot), $q=0.9$ in the range $0 \leqslant \alpha \leqslant 0.2$ (middle plot), and $q=0.95$ in the range $0 \leqslant \alpha \leqslant 0.225$ (bottom plot). The lightly shaded regions indicate where positive and negative polarity solitary waves can be found.

Eq. (15). The limit as $q \rightarrow 1$ (the Cairns limit) is $M_{\min }=$ $1 / \sqrt{1-\frac{4 \alpha}{1+3 \alpha}}$, which is in agreement with the value found in Ref. [14], Eq. (6), if we substitute $\beta=4 \alpha /(1+3 \alpha)$ therein.

The Maxwellian limit as $q \rightarrow 1, \alpha \rightarrow 0$ is $M_{\min }=1$, because we have normalized with respect to the Maxwellianbased ion acoustic speed $c_{s}$.

Figure 6 shows how the minimum Mach number $M_{\min }$ varies with $q$ in the range $0.6<q \leqslant 1$, for several values of $\alpha$, and we can see that there is a minimum value of $q$ for each corresponding value of $\alpha$ according to the condition we found in Sec. II: $\alpha \leqslant(2 q-1) / 4$. For example, at $\alpha=0.24$ (brown continuous line on the figure), the corresponding minimum value of $q$ is 0.98 . It is clear from this figure that the minimum
Mach speed is higher at lower values of $q$ and increases with increasing $\alpha$ (although the valid range for solitons diminishes as $\alpha$ is increased). This is also evident in Fig. 7, which shows the variation of $M_{\min }$ with $\alpha$ for different $q$. The steeper gradients at lower values of $q$ illustrate the fact that the acoustic speed is more sensitive to changes in $\alpha$ for smaller $q$.

\section{UPPER LIMIT OF MACH NUMBER $M$ (INFINITE COMPRESSION POINT)}

The upper limit of the Mach number $M\left(M_{\max }\right)$ can be found by imposing the condition

$$
\Psi\left(\phi_{m}, M\right) \geqslant 0,
$$

where $\phi_{m}=M_{\max }^{2} / 2$ is the maximum positive value of $\phi$ for which the ion density $n$ is real. The ion density is real for any negative value of $\phi$, and so, in this model, there is no maximum Mach number for negative polarity solitons. However, we should note that, using the Cairns-Tsallis model, we assume that because the electron thermal speed is far greater than typical ion acoustic motions, the electron fluid inertia can be ignored, and in this context, the electrons are essentially massless $[4,35,36]$. Corrections for finite electron mass would impose a cutoff for negative potential solitons at large values of $M$ and $\phi$.

Figure 8 shows $M_{\max }$ and $M_{\min }$ versus $\alpha$ for values of $q$, for the valid ranges of $\alpha$ as discussed in Sec. II. We can see that there is a window of opportunity for both positive and negative solitary waves to exist between the minimum and maximum Mach values for a given combination of $\alpha$ and $q$, but in the region above the $M_{\max }$ curve, only negative polarity solitary wave excitations are possible. In the region below the $M_{\text {min }}$ curve, the conditions are below the minimum Mach speed required for solitary waves.

\section{CONCLUSION}

We have investigated the properties of the hybrid CairnsTsallis distribution function introduced by Tribeche et al. [33] in the context of ion acoustic soliton behavior in plasmas with excess superthermal particles. It was found that great care must be taken with the use of this distribution, as the function itself diverges for large $|v|$ for $q \leqslant 0.5$, and the normalizing integral diverges for $q \leqslant 0.6$. Thus, the normalization constant, and hence the electron density $n_{e}(\phi)$, is positive and finite in only a very limited range of the nonextensivity parameter $q$, viz., $q>0.6$. However, there is a further restriction, in that for $q>1$, the distribution exhibits a thermal cutoff and is thus inappropriate for the consideration of plasmas with enhanced non-Maxwellian tails, thus reducing the range of interest to $0.6<q \leqslant 1$ only.

Furthermore, the physical requirement of having a monotonic distribution function to obviate the occurrence of kinetic instability imposes a further $(q, \alpha)$ constraint, the limits of which are given by $q=(2 \alpha-1) / 4$.

Using the Sagdeev method to find the associated pseudopotential for the valid ranges of $q$ and $\alpha$, we have evaluated the appropriate minimum and maximum Mach numbers to find existence conditions for ion acoustic solitary waves within the range of validity of the distribution function. 
Using this model, we see that some interesting solitary wave structures are possible, having either enhanced or depleted density, and coexistence of positive and negative potential solitons may occur, as is the case for the pure Cairns distribution, but not for the pure Tsallis distribution.

By introducing a further fitting parameter as compared to the pure Cairns, pure Tsallis and $\kappa$ distributions, it might appear that the product Cairns-Tsallis distribution could potentially be applicable to a wider range of situations involving high-energy non-Maxwellian tails. However, as we have seen, it is severely restricted in its applicability, as it is limited to the range of values of the nonextensive parameter $q$ satisfying $0.6<q \leqslant 1$, subject to the further constraint given by $q=(2 \alpha-1) / 4$.

Regrettably, it thus follows that the hybrid Cairns-Tsallis distribution does not add significantly to our understanding of the behavior of acoustic solitons in plasmas with distribution functions having an excess of superthermal particles in nonMaxwellian "tails," as are often observed in both space and the laboratory.

\section{ACKNOWLEDGMENTS}

The authors warmly acknowledge the referees for helpful comments. G.W. gratefully acknowledges funding from DEL NI (Department of Employment and Learning Northern Ireland) in the form of a Ph.D. studentship. I.K. acknowledges financial support from the UK Engineering and Physical Science Research Council (EPSRC) under Grant No. EP/I031766/1 ("Fluid Theory and Simulation for Laser-Plasma Interactions"). M.A.H. is grateful to the South African National Research Foundation for partial funding of this work.
[1] E. Infeld and G. Rowlands, Nonlinear Waves, Solitons and Chaos (Cambridge University Press, Cambridge, 2000).

[2] R. Z. Sagdeev, in Reviews of Plasma Physics, edited by M. A. Leontovich, Vol. 4 (Consultants Bureau, New York, 1966), p. 23.

[3] A. A. Mamun, Phys. Rev. E 55, 1852 (1997).

[4] F. Chen, Introduction to Plasma Physics and Controlled Fusion, Vol. 1 (Springer Science, Plenum, New York, 1984).

[5] L. Romagnani, J. Fuchs, M. Borghesi, P. Antici, P. Audebert, F. Ceccherini, T. Cowan, T. Grismayer, S. Kar, A. Macchi, P. Mora, G. Pretzler, A. Schiavi, T. Toncian, and O. Willi, Phys. Rev. Lett. 95, 195001 (2005).

[6] M. Borghesi, J. Fuchs, S. V. Bulanov, A. J. Mackinnon, P. K. Patel, and M. Roth, Fusion Sci. Technol. 49, 412 (2006).

[7] L. Romagnani, S. V. Bulanov, M. Borghesi, P. Audebert, J. C. Gauthier, K. Lowenbruck, A. J. Mackinnon, P. Patel, G. Pretzler, T. Toncian, and O. Willi, Phys. Rev. Lett. 101, 025004 (2008).

[8] R. A. Cairns, A. A. Mamun, R. Bingham, R. Boström, R. O. Dendy, C. M. C. Nairn, and P. K. Shukla, Geophys. Res. Lett. 22, 2709 (1995).

[9] A. A. Mamun, Eur. Phys. J. D 11, 143 (2000).

[10] César A. Mendoza-Briceño, S. M. Russel, and A. A. Mamun, Planet. Space Sci. 48, 599 (2000).

[11] X. Jukui, Chaos Solitons Fractals 18, 849 (2003).

[12] S. Maharaj, S. Pillay, R. Bharuthram, R. Reddy, S. Singh, and G. Lakhina, J. Plasma Phys. 72, 43 (2006).

[13] F. Verheest and S. Pillay, Phys. Plasmas 15, 013703 (2008).

[14] F. Verheest and M. A. Hellberg, Phys. Plasmas 17, 102312 (2010).

[15] T. K. Baluku and M. A. Hellberg, Plasma Phys. Controlled Fusion 53, 095007 (2011).

[16] C. Tsallis, J. Stat. Phys. 52, 479 (1988).

[17] R. Silva Jr., A. Plastino, and J. Lima, Phys. Lett. A 249, 401 (1998).

[18] J. A. S. Lima, R. Silva Jr., and Janilo Santos, Phys. Rev. E 61, 3260 (2000).
[19] I. Dubinova and A. Dubinov, Tech. Phys. Lett. 32, 575 (2006).

[20] L. Liu and J. Du, Physica A 387, 4821 (2008).

[21] Z. Liu, L. Liu, and J. Du, Phys. Plasmas 16, 072111 (2009).

[22] M. Tribeche, L. Djebarni, and R. Amour, Phys. Plasmas 17, 042114 (2010).

[23] B. Sahu, Phys. Plasmas 18, 082302 (2011).

[24] P. Eslami, M. Mottaghizadeh, and H. Pakzad, Phys. Plasmas 18, 102303 (2011).

[25] M. Bacha, M. Tribeche, and P. K. Shukla, Phys. Rev. E 85, 056413 (2012).

[26] M. Tribeche, L. Djebarni, and H. Schamel, Phys. Lett. A 376, 3164 (2012).

[27] V. Vasyliunas, J. Geophys. Res. 73, 2839 (1968).

[28] M. A. Hellberg, R. L. Mace, T. K. Baluku, I. Kourakis, and N. S. Saini, Phys. Plasmas 16, 094701 (2009).

[29] I. Kourakis, S. Sultana, and M. A. Hellberg, Plasma Phys. Controlled Fusion 54, 124001 (2012).

[30] M. Hapgood, C. Perry, J. Davies, and M. Denton, Planet. Space Sci. 59, 618 (2011).

[31] G. Livadiotis and D. J. McComas, J. Geophys. Res. Space Phys. 114, A11 (2009).

[32] G. Livadiotis and D. J. McComas, Astrophys. J. 741, 88 (2011).

[33] M. Tribeche, R. Amour, and P. K. Shukla, Phys. Rev. E 85, 037401 (2012).

[34] R. Amour, M. Tribeche, and P. Shukla, Astrophys. Space Sci. 338, 287 (2012).

[35] N. Krall and A. W. Trivelpiece, Principles of Plasma Physics (McGraw-Hill, New York, 1973).

[36] R. J. Goldston and P. H. Rutherford, Introduction to Plasma Physics (Institute of Physics, Bristol, 2003).

[37] We wish to thank a referee for pointing out that an even more stringent lower limit of (5/7) follows if one considers the need for a finite energy integral. As we wish to concentrate on the density only, we shall retain the more generous lower limit of 0.6 . 